\title{
Improving Compliance of Physicians in Reporting and Documenting Critical Alerts in a Cancer Hospital
}

\author{
Wania Imtiaz' ${ }^{1}$ Khawaja Shehryar Nasir ${ }^{1,2}$, Fareeha Kanwal², Sheeba Saqib², \\ Haroon Hafeez ${ }^{1,2}$
}

'Department of Internal Medicine, Shaukat Khanum Memorial Cancer Hospital and Research Centre, Lahore, Punjab, Pakistan, ${ }^{2}$ Department of Quality and Patient Safety, Shaukat Khanum Memorial Cancer Hospital and Research Centre, Lahore, Punjab, Pakistan

Received: 05 June 2021/Accepted: 30 June 2021

\section{OPEN ACCESS Correspondence: \\ Haroon Hafeez, Department of Internal Medicine, Shaukat Khanum Memorial Cancer Hospital and Research Centre, Lahore, Punjab, Pakistan. E-mail: haroonh@skm.org.pk \\ Citation: Imtiaz W, Nasir KS, Kanwal F, Saqib S, Hafeez H. Improving Compliance of Physicians in Reporting and Documenting Critical Alerts in a Cancer Hospital. $\mathrm{J}$ Cancer Allied Spec [Internet]. 2021 May $31 ; 7(2) . x x x$. https://doi.org/10.37029/jcas. v7i2.419}

Copyright: (c) 2021 Imtiaz, et al. This is an open access article distributed under the terms of the Creative Commons Attribution License, which permits unrestricted use, distribution, and reproduction in any medium, provided the original author and source are credited.

Funding: The authors received no financial support for the research, authorship and/or publication of this article.

Competing interest: Nil.

\begin{abstract}
Introduction: A critical result of an investigation is considered a representation of a pathophysiological state deemed to be high risk or life threatening for the patient. Therefore, such results should be addressed in an appropriate and timely manner. Unfortunately, routine closed chart audits suggested that the compliance of physicians in documenting critical alerts in patient notes was poor. This prompted the hospital to conduct a continuous quality improvement (CQI) project to improve the physicians' compliance. Materials and Methods: A cause-and-effect analysis was conducted using a fishbone diagram to identify the reasons for poor compliance. Based on the analysis, several modifications were made, including, but not limited to, hospital-wide educational sessions on the standard operating procedures of receiving and documenting critical alerts for the physicians, daily audit of critical alerts to review the appropriateness of documentation and introduction of a new module in the hospital electronic medical record to acknowledge and document receiving critical alerts. Results: Before implementing the strategies to improve physicians' documentation compliance, the average compliance rate was $57 \%$ in April 2020, and the median compliance rate was 52\% (January 2020-April 2020). However, afterward, within a couple of months of implementing changes, the average compliance rate increased to $88 \%$. This improvement was sustained for the next 8 months (median of $89 \%$ ). Conclusion: This study found that CQI approach can be used to improve the compliance of the physicians for appropriately and timely documenting critical alerts, in this case, by continued education and training process and incorporating changes into the electronic hospital information system.
\end{abstract}

Key words: Continuous quality improvement, critical alerts, documentation, investigations, optimisation of care

\section{Introduction}

Shaukat Khanum Memorial Cancer Hospital and Research Centre (SKMCH and RC) is a 195-bed state-of-the-art comprehensive cancer centre. The hospital provides oncological and allied services in outpatient and inpatient settings. In 2020 , the hospital had roughly 200,000 outpatient 
encounters and 11,000 admissions, and thousands of patients underwent surgical procedures, chemotherapy sessions and radiation treatments. As a result, nearly 4.7 million pathology tests and 141,000 radiology studies were conducted..$^{[1]}$

The Joint Commission on Accreditation of Healthcare Organizations deems a test result to be critical if there is a variance from the normal range representing a pathophysiologic state that is high risk or life threatening for the patient considered urgent or emergent, and immediate medical action is likely necessary to preserve life or prevent a catastrophic occurrence. This is different from an abnormal test result. In the latter, the results are outside of the expected range, but the outcome is not regarded as urgent or emergent and is less likely to be a life threat. ${ }^{[2,3]}$ Hence, immediate notification of a critical value to the appropriate healthcare professional is vital for patient care. The act of sharing a critical result with a physician is called generating a critical alert. One can argue that the significance of such results manifolds in vulnerable patient populations, such as those with an oncological disease or immunosuppression.

This study aimed to improve patient care by introducing structural and organisational improvements that would enhance the compliance of physicians in reporting and documenting critical alerts in a tertiary care cancer hospital.

\section{Materials and Methods}

This case study was conducted at $\mathrm{SKMCH}$ and RC, Lahore, Pakistan, from April 2020 to March 2021. It was instituted because, in April 2020, the documentation of receiving a critical alert by physicians was found to be $57 \%$ (326/572). The hospital Quality and Patient Safety Department conducted a quality improvement project to improve the process of documentation. A PlanDo-Check-Act (PDCA) was performed. In the first phase, with the help of senior residents from the department of internal medicine, a cause-and-effect analysis was conducted on the data from the first quarter of 2020 using a fishbone diagram to identify the causes for the lack of compliance [Figure 1]. The most significant problems highlighted were the rapid turnaround of junior doctors, high workload, lack of training and education about the hospital policies, poor documentation and infrequent audits of the process.

A series of training and educational sessions on hospital rules and policies, focusing on standard operating procedures (SOP) of receiving and documenting critical alerts, were arranged for the doctors across the hospital. Furthermore, this training and educational material was made part of the orientation program of the new joining physicians.

A daily audit of a randomised sample of 128 critical alerts was started. If there were any documentation errors, the healthcare worker involved was informed, educated and retrained. In addition, audit reports were shared weekly with the concerned head of departments.

The hospital uses an electronic hospital information system (HIS) for electronic documentation of medical records. This module ensured that an alert was generated automatically to the ordering physician if the laboratory test value fell in the critical range. Similarly, the healthcare staff, such as a radiologist or a respiratory therapist, was prompted by the system to declare if the test value was critical (if results were being manually entered in the system). Once reported, an automated email was sent to the ordering consultant notifying them of the test value. Furthermore, the healthcare staff, such as laboratory technicians, radiologists or respiratory therapists, called the on-call or the ordering physician, notified them and documented this on the HIS. In addition, the physician received a notification on their login screen of the HIS to enter details of the critical alert, including details of any actions that were taken. This ensured that critical alerts were generated, collected and acknowledged in an appropriate and timely manner. 


\section{Results}

In April 2020, 572 critical alerts were conveyed to physicians. However, the compliance of physician adequately documenting these alerts in the HIS was $57 \%$ [Figure 2]. Before the implementation of the quality improvement project, the median of physician documentation of critical alerts was $52 \%$ (January 2020-April 2020).
The hospital-wide educational sessions on the hospital policies and SOP of receiving and documenting critical alerts of the junior doctors were initiated in May 2020. Furthermore, this educational module was added in the new joining physician orientation. Likewise, in May 2020, a daily audit of a randomised sample of 128 charts in which critical alerts were generated was initiated. The discrepancies found were dealt with in real

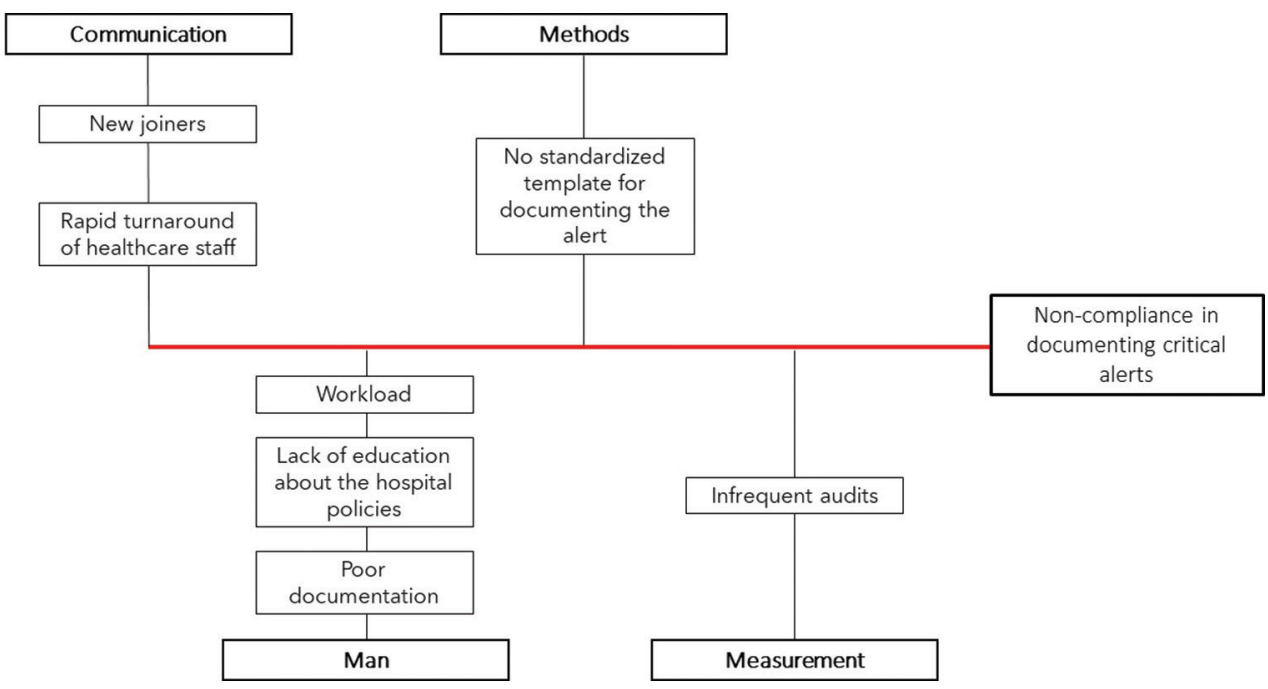

Figure 1: The documentation of critical alerts process flow highlighting the variable that contributes to inadequate documentation

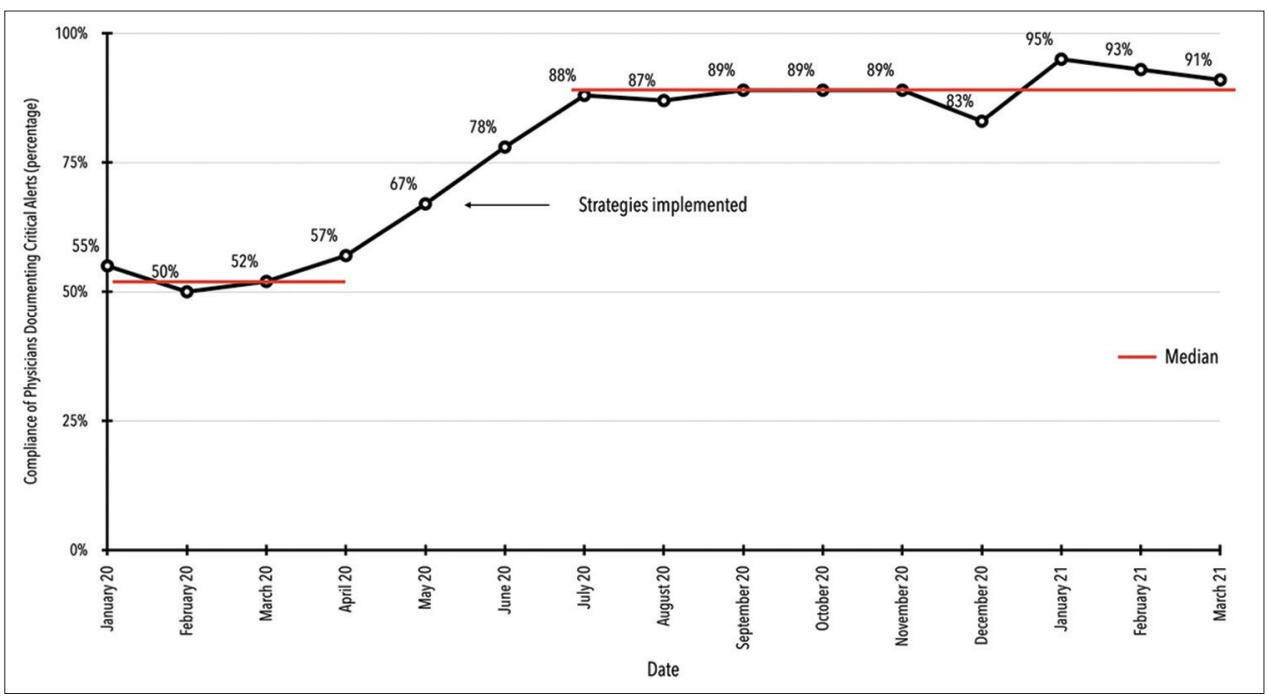

Figure 2: Average compliance of physicians for documenting critical alerts in the patient record during the study period. Before strategies were implemented, the median compliance rate was $52 \%$, and afterward, this increased to $89 \%$ 
time by contacting the physician team involved and counselling them. The results of these audits were shared weekly with department leaders. Altogether, this helped to improve the compliance of the physicians to $78 \%$ in June 2020.

In July 2020, a module was launched in the HIS to improve the timeliness and appropriateness of documentation of the critical alerts. This helped increase compliance to $88 \%$ by the end of the month. This progress gradually improved to $95 \%$ in January 2021. Post-implementing the quality improvement project, the median compliance of physician documentation increased to $89 \%$ (April 2020-March 2021).

\section{Discussion}

Continuous quality improvement (CQI) is a structured organisational process involving healthcare staff in planning and implementing proactive and reactive improvements in care processes to enhance qualitative healthcare outcomes. It aims to optimise clinical care by reducing variability and costs, enabling adherence to standardised clinical guidelines and improving the service quality. ${ }^{[3-6]}$

In this case study, the compliance of physicians in documenting critical alerts was found to be poor. However, with the structural and operational changes formulated through the COl project, physicians' compliance in documenting alerts rose to $95 \%$ at one point from a median of $52 \%$.

$\mathrm{CQI}$ projects can be executed in small- and largescale hospitals. There are examples in the literature where healthcare facilities were able to improve efficiency and optimise clinical flow by simplifying the information flow process, removing barriers or friction or by incorporating technology into the process. ${ }^{[4,6-8]}$ Similarly, it has been reported that formally organised CQI strategies often have an immediate impact on clinical practice. ${ }^{[9]}$ This was also observed in the present study. The compliance rate of documentation rose to $67 \%$ from $57 \%$ within 1 month of implementation of strategies. However, the sustainability of the modifications to the procedures after their initial implementation tends to degenerate. This has mainly been observed when monitoring or facilitating authorities leave the project. ${ }^{[8]}$ On the contrary, in the present study, a gradual and sustained improvement in compliance was observed. This is likely due to the robust changes made in the system operations, such as introducing a module in the HIS and the continued education and training activities.

The CQI approach can be used to improve the compliance of the physicians for appropriately and timely documenting critical alerts. Furthermore, this improvement can be sustained by introducing robust changes in the system operations.

\section{References}

1. Shaukat Khanum Memorial Cancer Hospital and Research Centre Facts and Statistics. Shaukat Khanum Memorial Cancer Hospital and Research Centre; 2021. Available from: https://www.shaukatkhanum. org.pk/about-us/facts-and-statistics. [Last accessed on 2021 Jun 17].

2. Joint Commission International, Joint Commission on Accreditation of Healthcare Organizations. Joint Commission International Accreditation Standards for Hospitals. Oakbrook Terrace, III: Joint Commission International; 2007.

3. Anthony SG, Prevedello LM, Damiano MM, Gandhi TK, Doubilet PM, Seltzer SE, et al. Impact of a 4-year quality improvement initiative to improve communication of critical imaging test results. Radiology 2011;259:802-7.

4. McCalman J, Bailie R, Bainbridge R, McPhail-Bell K, Percival N, Askew D, et al. Continuous quality improvement and comprehensive primary health care: A systems framework to improve service quality and health outcomes. Front Public Health 2018;6:76.

5. Maguerez G, Erbault M, Terra JL, Maisonneuve H, Matillon Y. Evaluation of 60 continuous quality improvement projects in French hospitals. Int J Qual Health Care J Int Soc Qual Health Care 2001;13:89-97.

6. Khan N, Hafeez H, Khawaja SN. Improving the efficiency and reducing variability in patient flow in an outpatient parenteral antibiotic therapy unit of a tertiary care hospital. Future Healthc J 2021.

7. Psaltikidis EM, da Silva EN, Moretti ML, Trabasso P, Stucchi RS, Aoki FH, et al. Cost-utility analysis of outpatient parenteral antimicrobial therapy (OPAT) in the Brazilian national health system. Expert Rev 
Pharmacoecon Outcomes Res 2019;19:341-52.

8. Geboers $H$, van der Horst M, Mokkink $H$, van Montfort $P$, van den Bosch W, van den Hoogen $H$, et al. Setting up improvement projects in small scale primary care practices: Feasibility of a model for continuous quality improvement. Qual Health Care OHC 1999;8:36-42.

9. Hogg S, Roe Y, Mills R. Implementing evidencebased continuous quality improvement strategies in an urban aboriginal community controlled health service in South East Queensland: A best practice implementation pilot. JBI Database Syst Rev Implement Rep 2017;15:178-87.

\section{Authorship Contributions}

Conceived and designed the analysis: WI, SS, KSN and HH. Collected the data: WI, FK and SS. Contributed data or analysis tools: SS and FK. Performed the analysis: KSN. Wrote the paper: WI, $\mathrm{KSN}$ and $\mathrm{HH}$. 